\title{
Effect of acute ethanol administration on zebrafish tail beat motion
}

\author{
Tiziana Bartolini ${ }^{1}$, Violet Mwaffo ${ }^{1}$, Sachit Butail ${ }^{2}$, Maurizio Porfiri ${ }^{1 *}$ \\ ${ }^{I}$ Department of Mechanical and Aerospace Engineering, New York University Polytechnic School of \\ Engineering, Brooklyn, New York, 11201, USA \\ ${ }^{2}$ Indraprastha Institute of Information Technology Delhi (IIITD), New Delhi 110020, India \\ *Corresponding author: mporfiri@nyu.edu, 718-260-3681 (phone), 718-260-3532 (fax)
}

\begin{abstract}
Zebrafish is becoming a species of choice in neurobiological and behavioral studies of alcohol-related disorders. In these efforts, the activity of adult zebrafish is typically quantified using indirect activity measures that are either scored manually or identified automatically from the trajectory of the fish centroid. The analysis of such activity measures has enabled important insight into the effect of acute ethanol exposure on individual and social behavior of this vertebrate species. Here, we leverage a recently developed tracking algorithm that reconstructs fish body shape to investigate the effect of acute ethanol administration on zebrafish tail beat motion, in terms of amplitude and frequency. Our results demonstrate a significant effect of ethanol on the tail beat amplitude as well as the tail beat frequency, both of which were found to robustly decrease for high ethanol concentrations. Such a direct measurement of zebrafish motor functions is in agreement with evidences based on indirect activity measures, offering a complementary perspective in behavioral screening.
\end{abstract}

Keywords: Ethanol; Locomotion; Tail beat amplitude; Tail beat frequency; Zebrafish. 


\section{Introduction}

Zebrafish is increasingly used as an animal model to study the effect of ethanol administration on individual and social behavior (Echevarria, Toms, \& Jouandot, 2011). In these experiments, zebrafish behavior is typically studied using measures of activity that are scored manually or computed automatically from the trajectory of the fish centroid. While previous experiments on acute ethanol treatment have highlighted significant effects on adult zebrafish locomotion (Blazina, Vianna, \& Lara, 2013; Cianca, Bartolini, Porfiri, \& Macrì, 2013; Gerlai, Lahav, Guo, \& Rosenthal, 2000; Ladu, Butail, Macrì, \& Porfiri, 2014; Mathur \& Guo, 2011; Spinello, Macrì, \& Porfiri, 2013), a lack of high-throughput methods to measure zebrafish tail beat motion has hindered a thorough understanding of the modalities in which ethanol impinges on motor functions.

Motor functions have been extensively investigated in larval zebrafish in terms of tail beat motion, during escape and predatory avoidance, prey capture, and swimming (Ikeda et al., 2013; McClenahan, Troup, \& Scott, 2012; Pham et al., 2012; Thorsen, Cassidy, \& Hale, 2004; Trivedi \& Bollmann, 2013; Zhou, Cattley, Cario, Bai, \& Burton, 2014), yet, studies on motor functions on adult zebrafish are rare. In prior studies on adult zebrafish, fish activity was investigated through the analysis of time spent swimming (Blazina et al., 2013; Cianca et al., 2013; Spinello et al., 2013), time spent freezing (Cianca et al., 2013; Gerlai, Lee, \& Blaser, 2006; Spinello et al., 2013), distance travelled (Gerlai, Ahmad, \& Prajapati, 2008; Gerlai et al., 2006), average speed (Ladu et al., 2014; Mathur \& Guo, 2011), and number of segment crossed in the tank (Gerlai et al., 2000; Maximino, da Silva, Gouveia, \& Herculano, 2011). While these measures are valid indicators of zebrafish activity and have been shown to be modulated by ethanol treatment, they should be considered as indirect, rather than direct measures of motor functions.

Acute ethanol exposure has been shown to induce changes in zebrafish locomotion, with higher locomotor activity at low concentrations and, vice versa, lower activity at high concentrations (Blazina et al., 2013; Cianca et al., 2013; Gerlai et al., 2000; Ladu et al., 2014; Maximino et al., 2011; Spinello et al., 
2013). The time spent swimming by fish in a spinning tank has been found to decrease at low ethanol concentrations (0.25\%) (Blazina et al., 2013), while similar doses have been observed to play a secondary role in preference tests conducted in placid water (Cianca et al., 2013; Spinello et al., 2013). Ethanol administration also modulates the time spent freezing by fish in placid water (Cianca et al., 2013; Spinello et al., 2013), whereby subjects treated at high ethanol concentrations (1.00\%) exhibit enhanced freezing response. Additionally, for experiments in placid water, both the number of crossings between different segments of the experimental tank (Gerlai et al., 2000; Maximino et al., 2011) and the average speed (Ladu et al., 2014) were found to display a U-shaped curve, with both these quantities being highest at low ethanol concentrations. While studies on zebrafish tail beat motion are scarce, experimental observations on several aquatic species have demonstrated a positive correlation between swimming speed, or distance travelled, and both the tail beat frequency and tail beat amplitude across a wide range of physical scales, from larvae to whales (Bainbridge, 1958; Gazzola, Argentina, \& Mahadevan, 2014).

Through a novel automated tracking algorithm capable of reconstructing fish body shape, we sought to elucidate the effects of acute ethanol treatment on the tail beat amplitude and frequency of adult zebrafish. We studied zebrafish locomotion in placid water for different ethanol treatment levels $(0.00 \%$, $0.25 \%, 0.50 \%$, and $1.00 \%$ ), using a high-resolution camera. Based on previous observations on the effect of ethanol on speed and the influence of the tail beat motion on the speed, we predicted that the tail beat frequencies and amplitude of zebrafish would be modulated by ethanol concentration. In line with previous studies on the effect of alcohol on zebrafish activity (Echevarria et al., 2011), we expected that one or both tail beat frequency and amplitude will increase at low concentrations $(0.25 \%)$ before registering a decrease at high concentration $(1.00 \%)$.

\section{Material and Methods}

\section{Ethics statement}

The experimental procedure described in this study was approved by the Animal Welfare Oversight Committee of New York University under protocol number 13-1424. 


\section{Animals and housing}

A total of 48 adult zebrafish (Danio rerio) of wild-type variety, approximately $3 \mathrm{~cm}$ in body length, were bought from an on-line aquarium vendor (LiveAquaria.com, Rhinelander, WI, USA) and housed in 37.8 liter (10 gallon) tanks with a maximum stocking density of 0.5 fish per liter. Temperature and $\mathrm{pH}$ were maintained at $25^{\circ} \mathrm{C} \pm 1^{\circ} \mathrm{C}$ and 7.2 respectively. The fish were kept on a 14 hours light and 10 hours dark cycle according to the circadian rhythm of this species (Cahill, 2002; Matthews, Trevarrow, \& Matthews, 2002). Experiments were initiated after a 15-days habituation period, and fish were fed daily after the experimental session with commercial flake food between 5-6 pm each day.

\section{Apparatus}

The experimental tank measured $30 \times 10 \times 15 \mathrm{~cm}$ (length $\times$ breadth $\times$ height). The tank itself was positioned within a larger square tank, $120 \mathrm{~cm}$ in length and was covered with a white contact paper in order to avoid reflections and provide a high-contrast background for visual tracking. A high-resolution camera (Flea 3 USB 3.0, PointGrey Research, Canada) was mounted $20 \mathrm{~cm}$ directly above the tank; the height of the camera was adjusted to ensure maximum coverage of the experimental tank. Four fluorescent tubes of $25 \mathrm{~W}$ each (All-Glass Aquarium, UK) were mounted on the each side of the square tank. The setup was surrounded by dark curtains to visual isolate the apparatus.

\section{Drugs and treatments}

The effect of ethanol administration was tested with four conditions corresponding to the following concentrations: $0.00 \%$ (control, equivalent to (Mwaffo, Butail, di Bernardo, \& Porfiri, 2015)), 0.25\%, $0.50 \%$, and $1.00 \%$. Absolute EtOh (200 proof) used in the experiment was purchased from Fisher Scientific (Fair Lawn, NJ, USA). Each focal fish was transferred with a hand net from the holding tank to a $500 \mathrm{ml}$ glass beaker filled with water from the experimental tank and treated with the corresponding ethanol concentration, $0 \%(0 \mathrm{mM}), 0.25 \%(43 \mathrm{mM}), 0.50 \%(86 \mathrm{mM})$, and $1 \%(171 \mathrm{mM})$. The ethanol solution in the beaker was changed for each fish, which was kept in the beaker for one hour before being 
placed into the experimental tank. We expect these concentrations to be relatively stable during the one hour of exposure (Irons, MacPhail, Hunter, \& Padilla, 2010). After exposure to ethanol in the beaker, the fish was transferred with a hand net to the experiment tank, which had the same ethanol concentration of the beaker. The one-hour exposure time was selected to allow for the stabilization the blood alcohol levels in fish (Gerlai et al., 2006; R. Ryback, Percarpio, \& Vitale, 1969; R. S. Ryback, 1976).

\section{Experimental procedure}

The experimental session was performed in October 2014 and consisted of 12 trials $(n=12)$ for each ethanol concentration. Each trial consisted of two 60 seconds observations in the experimental tank; the first observation was recorded 30 seconds after the fish was placed in the tank and the second observation 10 minutes later (Gerlai et al., 2000). Experimental sessions were video-recorded at 60 frames per second at a resolution of $1280 \times 1024$ pixels. All videos were stored and analyzed offline with an in-house developed multi-target shape-tracking algorithm developed in prior work by our group (Bartolini, Butail, \& Porfiri, 2014).

\section{Behavioral observations}

For each trial, we measured the following quantities: time spent freezing, tail-beat frequency (TBF), tail-beat amplitude (TBA), and average speed. The time spent freezing was computed by dividing the videos into 2-second intervals and counting the number of intervals during which the position of the fish, measured using its centroid on the image, did not move beyond a circle of radius equal to $2 \mathrm{~cm}$ (Kopman, Laut, Polverino, \& Porfiri, 2013). The average speed was estimated from an optimally weighted difference of the fish centroid in successive frames, output from the tracking software (Bartolini et al., 2014).

Fish TBA and TBF were also estimated by the tracking algorithm (Bartolini et al., 2014), which fitted a parabola on the fish body in each video frame. Specifically, we considered the time series of the tail tip displacement with respect to the fish head. TBA and TBF were computed by identifying spikes in amplitude greater than a threshold of $0.6 \mathrm{~cm}$, exemplifying a tail flick in either direction. The minimum 
threshold value was set in accordance to the average fin length of the adult zebrafish used for this study. A maximum threshold was set at $1.5 \mathrm{~cm}$ to avoid false reading from the reconstruction of the tail tip position. The TBA was obtained by averaging the amplitude of these spikes over the whole length of the time series, instead of a selected window as in (Thomas \& Janz, 2011). The TBF was obtained as the frequency of these spikes in the video frames. If the fish was not moving in an entire video both TBF and TBA were set to zero.

\section{Statistical analysis}

Two factors ANOVA with replications was performed to study within-group variability, with time as the independent variable, and between-group variability across conditions, with ethanol concentration as the independent variable. A Levene's test for homoscedasticity of variances was used to evaluate whether the different ethanol concentrations elicited equally- or differentially variable interindividual tail beat motion (TBA and TBF). Fisher PLSD tests were used to in post-hoc analysis.

Significance level was set to $\mathrm{p}<0.05$.

\section{Results}

Ethanol administration influenced the time spent freezing by experimental subjects $\left(\mathrm{F}_{3,88}=4.70, \mathrm{p}\right.$ $<0.01)$; as well as the time $\left(\mathrm{F}_{1,88}=15.96, \mathrm{p}<0.01\right)$ and the ethanol $\times$ time interaction $\left(\mathrm{F}_{3,88}=4.48, \mathrm{p}<\right.$ 0.01), see Figure 1 (a). Specifically, the time spent freezing was significantly less in $0.00 \%$ for the first and the tenth minute compared to $1.00 \%$ ( $\mathrm{p}<0.05$ in post-hoc analysis). A significant effect of ethanol concentration $\left(\mathrm{F}_{3,88}=3.36, \mathrm{p}<0.05\right)$ and time $\left(\mathrm{F}_{1,88}=7.96, \mathrm{p}<0.01\right)$ was found on the average speed, see Figure $1(b)$. Ethanol $\times$ time interaction was instead non-significant $\left(F_{3,88}=0.90, p=0.44\right)$. While a $U$ shaped dependence of the average speed on ethanol administration in the first minute of the observation is suggested by Figure 1 (b), none of the post-hoc comparisons showed a significant difference between conditions (post-hoc comparisons between $1.00 \%$ fish and any other group yielded a probability value between 0.062 and 0.13 ). 
Figure 2 (a) displays a typical time trace for the tail tip displacement of control subjects, with a sequence of six alternating spikes identifying when the fish beats its tail. For clarity, in the inset we report the shape of the fish from the tracking software, displaying the body undulation for three selected frames, in which the fish bends its body upward, swims forward with a nearly flat body shape, and finally bends its body downward. The strokes are on the order of $1 \mathrm{~cm}$ and are separated by approximately $0.4 \mathrm{~s}$ (see Figure 2 (a)). Figure 2 (b) shows the corresponding speed, which widely ranges from 1 to $7 \mathrm{~cm} / \mathrm{s}$ as the fish bends its body, with the larger values in speed approximately following the tail beats.

Ethanol modulated both the TBF and TBA in the two minutes of observation. ANOVA on TBF revealed a significant ethanol effect on treated subjects $\left(F_{3,88}=4.22, p<0.01\right.$, Figure $\left.3(a)\right)$, but time $\left(F_{1,88}\right.$ $=0.002, \mathrm{p}=0.96)$ and ethanol $\times$ time $\left(\mathrm{F}_{3,88}=0.76, \mathrm{p}=0.52\right)$ interaction effects were non-significant. Specifically, in the tenth minute TBF of $1.00 \%$ subjects was significantly less than control individuals in post-hoc analysis. Similarly, TBA (Figure $3(\mathrm{~b})$ ) was also modulated by the ethanol concentration $\left(\mathrm{F}_{3,88}=\right.$ 9.7, $\mathrm{p}<0.01)$, yet, time $\left(\mathrm{F}_{1,88}=0.11, \mathrm{p}=0.75\right)$ and ethanol $\times$ time $\left(\mathrm{F}_{3,88}=0.15, \mathrm{p}=0.93\right)$ interaction effects were non-significant. Post-hoc analysis indicated that TBA of fish treated with the highest ethanol dose $(1.00 \%)$ was less than control subjects for both the first and the last minute of observation. Levene's test for homoscedasticity of variances indicated that TBF of individuals treated at $0.50 \%$ and $1.00 \%$ ethanol concentration was more variable than TBF of control subjects for the first minute of observation $(\mathrm{p}<0.05)$. Similarly, TBA of individuals treated at $0.50 \%$ and $1.00 \%$ concentrations was more variable than TBA of control subjects for both minute of observation $(\mathrm{p}<0.05)$. All the values used in the analysis are given in Table 1.

\section{Discussion}

Our results show that acute ethanol administration elicits robust changes in zebrafish behavior and locomotion. In agreement with prior studies (Cianca et al., 2013; Spinello et al., 2013), highly treated subjects spent more time freezing than control individuals. Such increase in freezing behavior was accompanied by a trend in the average speed, which suggested higher speed at low ethanol concentrations 
and lower speed at high concentrations in the first minute of observation similar to (Ladu et al., 2014; Mathur \& Guo, 2011). Both the tail beat amplitude and frequency were influenced by ethanol administration. Specifically, fish treated to high ethanol concentrations displayed smaller tail beat amplitude than control subjects during the first and tenth minute of observation. In addition, the tail beat frequency of subjects treated to high concentrations was less than control individuals in the tenth minute of the experiment.

The unavailability of computational tools to extract zebrafish body shape in previous experimental studies has hindered a systematic analysis of the tail beat motion. Thus, most of the literature on zebrafish behavioral phenotyping has focused on observable quantities related to the motion of the fish centroid, such as speed, turning rate, distance covered, and time budgeting in swimming and freezing. With growing access to high-resolution video recordings and fast image processing algorithms (Butail \& Paley, 2012; Fontaine et al., 2008; Pérez-Escudero, Vicente-Page, Hinz, Arganda, \& de Polavieja, 2014), zebrafish locomotion can now be studied by reconstructing the full body shape (Delcourt, Denoël, Ylieff, \& Poncin, 2013; Dell et al., 2014; Fontaine et al., 2008; Tian et al., 2014). In this work, we sought to implement automatic shape reconstruction to characterize the effect of ethanol administration on tail beat amplitude and frequency.

Our work seeks to complement existing experimental studies on the effect of ethanol on zebrafish locomotion, which have focused primarily on larvae (Ikeda et al., 2013; Irons et al., 2010; MacPhail et al., 2009). For example, by using the software developed in (MacPhail et al., 2009), (Ikeda et al., 2013) have demonstrated that subjects treated at high ethanol concentrations $(300 \mathrm{mM})$ exhibit a slightly lower tail beat than control subjects. Such a reduction in tail beat frequency is accompanied by a robust, twofold, increase in tail beat amplitude, resulting in considerably higher speed.

While adult zebrafish tail beat motion in response to ethanol administration has never been addressed, other studies have elucidated impaired swimming from toxicant-induced alterations, caused by 2,4-dinitrophenol (Marit \& Weber, 2011) and Selenomethionine (Se-Met) (Thomas \& Janz, 2011). These studies have demonstrated that zebrafish motor functions are modulated by the toxicant level, with higher 
doses leading to reduced tail beat motion. Different from our experimental setup, these efforts (Marit \& Weber, 2011; Thomas \& Janz, 2011) were conducted in a water channel, where focal subjects were forced to swim against a water current at a speed of several body length per second. As a result, tail beat frequencies observed therein are approximately five times larger than the values observed in our experiment, while the measured tail beat amplitude is approximately a half. In placid water, zebrafish do not beat their tail continuously and, instead, exhibit a burst-and-coast swimming style (Danos \& Lauder, 2007; Muller, Stamhuis, \& Videler, 2000). As shown in Figure 2, sporadic bursts of the tail are followed by coasting phases, during which zebrafish swim forward without beating their tail. We therefore process the time series of the relative tail-tip displacement to extract isolated peaks, whose amplitude and frequency are associated with the tail beat amplitude and frequency.

The reduction of tail beat frequency and amplitude at high ethanol concentration could be associated with slowness caused by the anesthetic-like sedative effects of alcohol (Gerlai et al., 2000). At high ethanol concentration, fish spent approximately half of the experimental time freezing. It is thus tenable to hypothesize that their tail beat motion during swimming was not sufficient to compensate for the complete inactivity during freezing. An alternative explanation could entail impaired swimming (Gerlai et al., 2000). At high ethanol concentration, fish were found to display a more variable tail beat motion, which could suggest reduced motor coordination.

Our results should be considered valid only for wild-type zebrafish, whose response to ethanol is investigated in this study. Experimental observations from other studies suggest that the effect of ethanol on zebrafish behavior is dependent upon the strain; for example wild-type zebrafish have been shown to be less sensitive to ethanol than long-fin striped (Dlugos \& Rabin, 2003). Thus, it is tenable to hypothesize that changing the strain will modulate the influence of ethanol on tail beat motion.

As anticipated from the literature on fish swimming (Bainbridge, 1958; Gazzola et al., 2014), the simultaneous reductions of the tail beat amplitude and frequency translated into a moderate average speed at high ethanol concentration. Indeed, the product of the tail beat amplitude and frequency is proportional to the so-called swimming number, which has been shown to be positively correlated to the average 
speed, with a functional relationship that is controlled by the flow regime. For turbulent flows, the swimming speed should be linearly proportional to the swimming number, while for laminar flows the scaling should have a power greater than one (Gazzola et al., 2014). Adult zebrafish swimming falls in between these two flow regimes, whereby the swimming number is between $10^{3}$ and $10^{4}$.

In conclusion, our study demonstrated the feasibility of assessing the effect of acute ethanol administration on zebrafish tail beat motion through an automated high-throughput tracking algorithm. Both the tail beat amplitude and the frequency, extracted from the time evolution of the fish body shape, were found to be modulated by ethanol concentration. These findings complement existing observations based on indirect measures of activity, such as the time spent swimming, time spent freezing, distance travelled, average speed, and number of segments crossed in the tank. Different from these indirect measures, the analysis of the tail beat motion offers a direct quantification of motor functions, confirming, for example, reduced locomotor activity at high ethanol concentrations. Future studies should focus on integrating the proposed methodology in established experimental paradigms, involving escape and predatory avoidance, prey capture, and sustained swimming against a water flow.

\section{Acknowledgements}

The authors gratefully acknowledge Fabrizio Ladu for his help with the experiments. This work was supported by the National Science Foundation under grant nos. CMMI-1129820, CMMI-1433670, and DGE-0741714 and the Mitsui USA foundation.

\section{References}

Bainbridge, R. (1958). The speed of swimming of fish as related to size and to the frequency and amplitude of the tail beat. Journal of Experimental Biology, 35(1), 109-133.

Bartolini, T., Butail, S., \& Porfiri, M. (2014). Temperature influences sociality and activity of freshwater fish. Environmental Biology of Fishes, 98(3), 825-832. 
Blazina, A. R., Vianna, M. R., \& Lara, D. R. (2013). The spinning task: A new protocol to easily assess motor coordination and resistance in zebrafish. Zebrafish, 10(4), 480-485.

Butail, S., \& Paley, D. A. (2012). Three-dimensional reconstruction of the fast-start swimming kinematics of densely schooling fish. Journal of the Royal Society Interface, 9(66), 77-88.

Cahill, G. M. (2002). Clock mechanisms in zebrafish. Cell and Tissue Research, 309(1), 27-34.

Cianca, V., Bartolini, T., Porfiri, M., \& Macrì, S. (2013). A robotics-based behavioral paradigm to measure anxiety-related responses in zebrafish. PLoS ONE, 8(7), e69661.

Danos, N., \& Lauder, G. V. (2007). The ontogeny of fin function during routine turns in zebrafish Danio rerio. Journal of Experimental Biology, 210(19), 3374-3386.

Delcourt, J., Denoël, M., Ylieff, M., \& Poncin, P. (2013). Video multitracking of fish behaviour: A synthesis and future perspectives. Fish and Fisheries, 14(2), 186-204.

Dell, A. I., Bender, J. A., Branson, K., Couzin, I. D., de Polavieja, G. G., Noldus, L. P., et al. (2014). Automated image-based tracking and its application in ecology. Trends in Ecology \&Evolution, 29(7), 417-428.

Dlugos, C. A., \& Rabin, R. A. (2003). Ethanol effects on three strains of zebrafish: model system for genetic investigations. Pharmacology Biochemistry and Behavior, 74(2), 471-480.

Echevarria, D. J., Toms, C. N., \& Jouandot, D. J. (2011). Alcohol-induced behavior change in zebrafish models. Reviews in the Neurosciences, 22(1), 85-93.

Fontaine, E., Lentink, D., Kranenbarg, S., Müller, U. K., van Leeuwen, J. L., Barr, A. H., et al. (2008). Automated visual tracking for studying the ontogeny of zebrafish swimming. Journal of Experimental Biology, 211(8), 1305-1316.

Gazzola, M., Argentina, M., \& Mahadevan, L. (2014). Scaling macroscopic aquatic locomotion. Nature Physics.

Gerlai, R., Ahmad, F., \& Prajapati, S. (2008). Differences in acute alcohol-induced behavioral responses among zebrafish populations. Alcoholism, Clinical and Experimental Research, 32(10), 17631773. 
Gerlai, R., Lahav, M., Guo, S., \& Rosenthal, A. (2000). Drinks like a fish: zebra fish (Danio rerio) as a behavior genetic model to study alcohol effects. Pharmacology Biochemistry and Behavior, 67(4), 773-782.

Gerlai, R., Lee, V., \& Blaser, R. (2006). Effects of acute and chronic ethanol exposure on the behavior of adult zebrafish (Danio rerio). Pharmacology Biochemistry and Behavior, 85(4), 752-761.

Ikeda, H., Delargy, A. H., Yokogawa, T., Urban, J. M., Burgess, H. A., \& Ono, F. (2013). Intrinsic properties of larval zebrafish neurons in ethanol. PloS ONE, 8(5), e63318.

Irons, T. D., MacPhail, R. C., Hunter, D. L., \& Padilla, S. (2010). Acute neuroactive drug exposures alter locomotor activity in larval zebrafish. Neurotoxicol Teratol, 32(1), 84-90.

Kopman, V., Laut, J., Polverino, G., \& Porfiri, M. (2013). Closed-loop control of zebrafish response using a bioinspired robotic-fish in a preference test. Journal of the Royal Society Interface, 10(78), 20120540.

Ladu, F., Butail, S., Macrì, S., \& Porfiri, M. (2014). Sociality modulates the effects of ethanol in zebra fish. Alcohol Clin Exp Res, 38(7), 2096-2104.

MacPhail, R., Brooks, J., Hunter, D., Padnos, B., Irons, T., \& Padilla, S. (2009). Locomotion in larval zebrafish: influence of time of day, lighting and ethanol. Neurotoxicology, 30(1), 52-58.

Marit, J. S., \& Weber, L. P. (2011). Acute exposure to 2, 4-dinitrophenol alters zebrafish swimming performance and whole body triglyceride levels. Comparative Biochemistry and Physiology Part C: Toxicology \& Pharmacology, 154(1), 14-18.

Mathur, P., \& Guo, S. (2011). Differences of acute versus chronic ethanol exposure on anxiety-like behavioral responses in zebrafish. Behavioural Brain Research, 219(2), 234-239.

Matthews, M., Trevarrow, B., \& Matthews, J. (2002). A virtual tour of the guide for zebrafish users. Resource, 31, 34-40.

Maximino, C., da Silva, A. W. B., Gouveia, A., \& Herculano, A. M. (2011). Pharmacological analysis of zebrafish (Danio rerio) scototaxis. Progress in Neuro-Psychopharmacology and Biological Psychiatry, 35(2), 624-631. 
McClenahan, P., Troup, M., \& Scott, E. K. (2012). Fin-tail coordination during escape and predatory behavior in larval zebrafish. PLoS ONE, 7(2), e32295.

Muller, U., Stamhuis, E., \& Videler, J. (2000). Hydrodynamics of unsteady fish swimming and the effects of body size: comparing the flow fields of fish larvae and adults. Journal of Experimental Biology, 203(2), 193-206.

Mwaffo, V., Butail, S., di Bernardo, M., \& Porfiri, M. (2015). Measuring Zebrafish Turning Rate. Zebrafish.

Pérez-Escudero, A., Vicente-Page, J., Hinz, R. C., Arganda, S., \& de Polavieja, G. G. (2014). idTracker: tracking individuals in a group by automatic identification of unmarked animals. Nature Methods, 11(7), 743-748.

Pham, M., Raymond, J., Hester, J., Kyzar, E., Gaikwad, S., Bruce, I., et al. (2012). Assessing social behavior phenotypes in adult zebrafish: shoaling, social preference, and mirror biting tests. In A. V. Kalueff \& A. M. Stewart (Eds.), Zebrafish Protocols for Neurobehavioral Research (Vol. 66, pp. 231-246): Humana Press.

Ryback, R., Percarpio, B., \& Vitale, J. (1969). Equilibration and metabolism of ethanol in the goldfish. Nature, 222(5198), 1068-1070.

Ryback, R. S. (1976). A method to study short-term memory (STM) in the goldfish. Pharmacology Biochemistry and Behavior, 4(4), 489-491.

Spinello, C., Macrì, S., \& Porfiri, M. (2013). Acute ethanol administration affects zebrafish preference for a biologically inspired robot. Alcohol, 47(5), 391-398.

Thomas, J., \& Janz, D. (2011). Dietary selenomethionine exposure in adult zebrafish alters swimming performance, energetics and the physiological stress response. Aquatic Toxicology, 102(1), 79-86.

Thorsen, D. H., Cassidy, J. J., \& Hale, M. E. (2004). Swimming of larval zebrafish: fin-axis coordination and implications for function and neural control. Journal of Experimental Biology, 207(24), 4175-4183. 
Tian, J., Satpathy, A., Ng, E. S., Ong, S. G., Cheng, W., Burgunder, J.-M., et al. (2014). Motion analytics of zebrafish using fine motor kinematics and multi-view trajectory. Multimedia Systems.

Trivedi, C. A., \& Bollmann, J. H. (2013). Visually driven chaining of elementary swim patterns into a goal-directed motor sequence: a virtual reality study of zebrafish prey capture. Frontiers in Neural Circuits, 7.

Zhou, Y., Cattley, R. T., Cario, C. L., Bai, Q., \& Burton, E. A. (2014). Quantification of larval zebrafish motor function in multiwell plates using open-source MATLAB applications. Nature Protocols, $9(7), 1533-1548$. 


\section{Figure Captions}

Figure 1. Average \pm standard error mean time spent freezing (a) and average speed (b) of the focal fish at different ethanol concentration. Curly braces identify significant differences in posthoc analysis.

Figure 2. Sample relative tail-tip displacement time series of zebrafish (a) and the corresponding speed (b). Peaks in the time series of the relative tail-tip displacement, seven in this dataset, represent fish tail strokes.

Figure 3. Average \pm standard error mean TBF (a) and TBA (b) of the focal fish at different ethanol concentrations. Curly braces identify significant differences in post-hoc analysis.

\section{Table Captions}

Table 4. Synoptic presentation of the main experimental results of the study. 
Figure 1

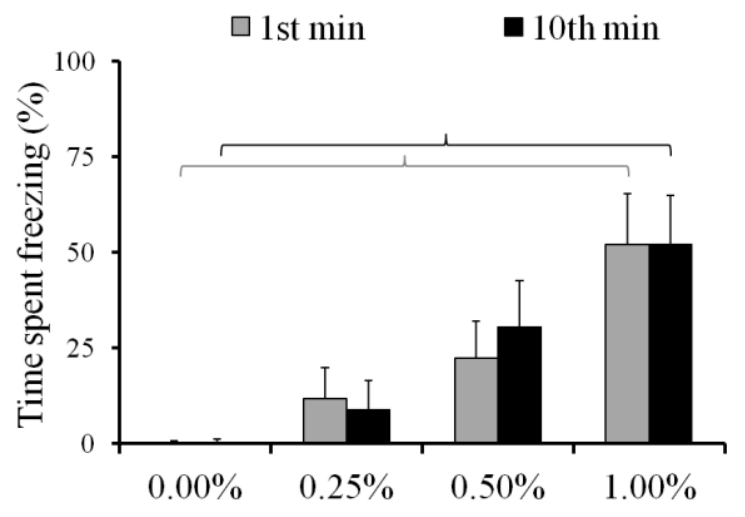

(a)

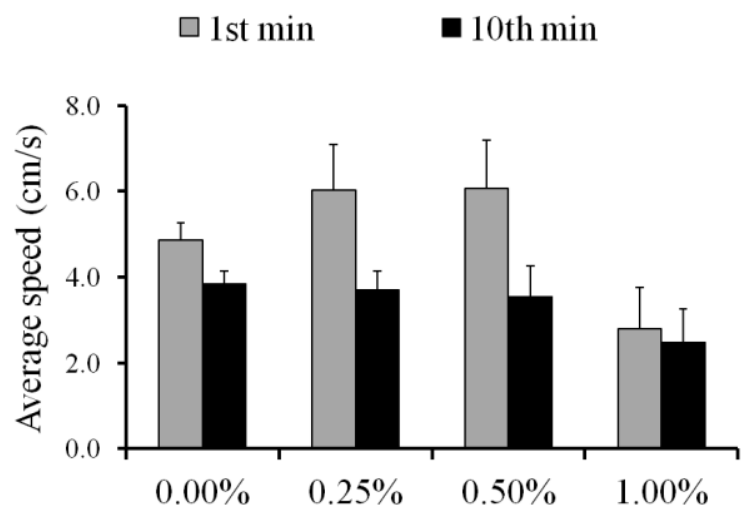

(b) 
Figure 2

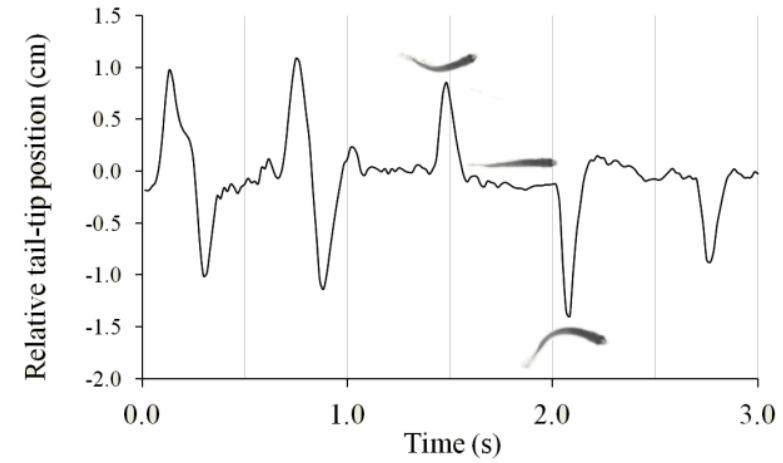

(a)

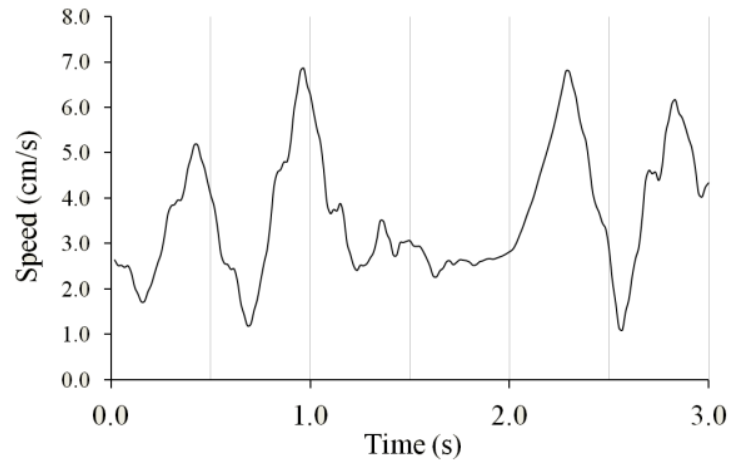

(b) 
Figure 3
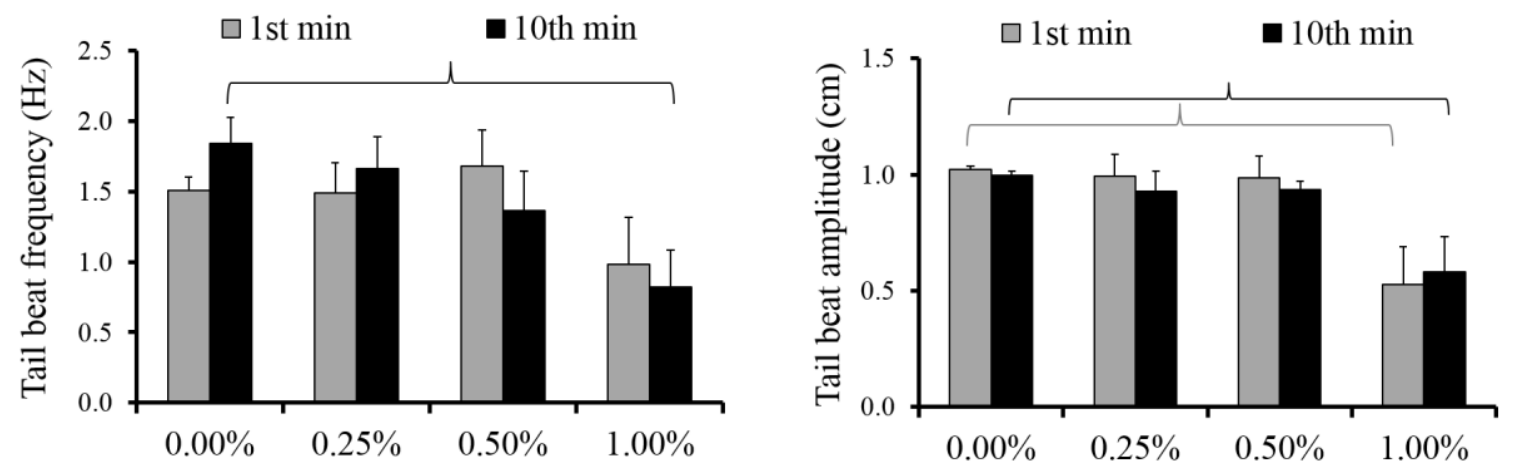

(a)

(b) 
Table 1

\begin{tabular}{|c|c|c|c|c|c|c|c|c|c|c|c|c|c|c|c|c|}
\hline & \multicolumn{4}{|c|}{ Time spent freezing (\%) } & \multicolumn{4}{|c|}{ Time spent swimming (\%) } & \multicolumn{4}{|c|}{ Tail beat frequency (Hz) } & \multicolumn{4}{|c|}{ Tail beat amplitude $(\mathrm{cm})$} \\
\hline & $1^{\text {st }} \min$ & & $10^{\text {th }} \mathrm{m}$ & & $1^{\mathrm{st}} \min$ & & $10^{\text {th }} \mathrm{m}$ & nute & $1^{\text {st }} \min$ & & $10^{\text {th }} \mathrm{m}$ & iute & $1^{\text {st }} \mathrm{min}$ & & $10^{\text {th }} \mathrm{mi}$ & ute \\
\hline & Mean & St. error & Mean & St. error & Mean & St. error & Mean & St. error & Mean & St. error & Mean & St. error & Mean & St. error & Mean & St. error \\
\hline $0.00 \%$ & 0.28 & 0.28 & 0.28 & 0.28 & 4.87 & 0.40 & 3.86 & 0.99 & 1.51 & 0.09 & 1.84 & 0.19 & 1.02 & 0.01 & 1.00 & 0.02 \\
\hline $0.25 \%$ & 11.67 & 8.22 & 8.89 & 7.72 & 6.06 & 1.04 & 3.72 & 1.51 & 1.49 & 0.21 & 1.66 & 0.22 & 0.99 & 0.09 & 0.93 & 0.09 \\
\hline $0.50 \%$ & 22.22 & 9.81 & 30.55 & 11.50 & 6.09 & 1.13 & 3.58 & 2.44 & 1.68 & 0.26 & 1.36 & 0.28 & 0.99 & 0.09 & 0.94 & 0.04 \\
\hline $1.00 \%$ & 51.94 & 13.44 & 52.22 & 12.90 & 2.91 & 0.96 & 2.58 & 2.63 & 0.98 & 0.34 & 0.82 & 0.26 & 0.53 & 0.16 & 0.58 & 0.15 \\
\hline
\end{tabular}

